\title{
Peritoneal dialysis patients - the forgotten group in the coronavirus pandemic
}

\author{
Authors: Laura Balson ${ }^{A}$ and Jyoti Baharani ${ }^{B}$
}

\begin{abstract}
While all patients with chronic disease have undoubtedly been affected by the ongoing SARS-CoV-2 (COVID-19) pandemic, individuals with end-stage renal failure have suffered significant excess morbidity and mortality. Patients on haemodialysis have received extensive research and media attention into their vulnerability to the disease; however, those receiving peritoneal dialysis (PD) have been much less visible. We surveyed a selection of patients from a cohort receiving PD at a tertiary renal unit in Birmingham, UK. We devised a questionnaire looking at patients' experience of shielding, accessing both dialysis and general medical care during the pandemic, and their thoughts about the pandemic and the future. Concerning findings were apparent from this. Attending hospital was the most commonly cited reason for being unable to shield, and multiple patients experienced difficulties accessing care while unwell during this period. Worryingly, $58 \%$ of respondents indicated that they feel negatively, or feel ambivalent, about the future. Patients receiving PD have suffered significantly during the COVID-19 pandemic and face ongoing difficulties and risks while accessing medical care. It is vital that this cohort is not forgotten in the planning of renal services during the pandemic, and that special attention is paid to both their physical and mental health.
\end{abstract}

KEYWORDS: dialysis, peritoneal dialysis, COVID-19, coronavirus, patient experience

DOI: 10.7861/clinmed.2021-0259

\section{Introduction and aim}

While all chronic disease patient groups have undoubtedly been affected by the ongoing SARS-CoV-2 (COVID-19) pandemic, it is now well known that individuals with end-stage renal failure have suffered significant excess morbidity and mortality over the past year. ${ }^{1}$ There are approximately 56,000 patients with end-stage renal failure in England, the majority of whom require renal replacement therapy in order to survive. ${ }^{2}$ Between March and

Authors: ${ }^{A}$ IMT3, University Hospitals Birmingham NHS Foundation Trust, Birmingham, UK; ${ }^{B}$ Consultant physician and nephrologist, University Hospitals Birmingham NHS Foundation Trust, Birmingham, UK
August 2020, 4,370 of these patients had laboratory-confirmed COVID-19 infection, and 1,291 of these subsequently died within 28 days of a positive COVID-19 result. ${ }^{2}$ This represents a startling mortality of around $29.5 \%$, which is far in excess of that of the general population in a similar time period. ${ }^{3}$ It is true that patients receiving in-centre haemodialysis (HD) are the worst affected, and also represent the largest cohort of end-stage renal failure patients. Consequently, patients on HD have received both academic and media attention in terms of their vulnerability to COVID-19, which is sadly exacerbated by time spent in busy dialysis centres where infection may unwittingly be spread. Patients receiving peritoneal dialysis (PD) have, however, been much less visible. This is despite the fact that they share many of the same clinical vulnerabilities as those undergoing HD, in terms of the immunocompromise associated with end-stage renal disease and the increased incidence of ischaemic heart disease and other cardiac complications. ${ }^{4}$ Approximately $12 \%$ of all dialysis patients in the UK are receiving PD. ${ }^{5}$

While scant research is emerging relating to the reorganisation and logistics of PD services during the pandemic, there are no UK studies focused on patient experience for those receiving PD during this period. Having noticed this gap in public and research interest, we hoped to capture the mood, opinions and experiences of this patient group in a questionnaire-based study. Our aim was to better understand how patients on PD have been affected by the pandemic thus far, and how renal services could better support them going forward.

\section{Methods}

We surveyed a cross-section of patients from a cohort receiving PD during the pandemic under the care of a tertiary renal unit in Birmingham, UK. During this time our service ran using national guidance from the National Institute for Health and Care Excellence (NICE) and other bodies, including providing shielding information to all of our patients and stopping any non-essential face-to-face contact. We focused on patients already established on PD for more than 3 months, as new starters often require more frequent contact with the service and have different needs. We devised a patient-reported experience measure (PREM) looking at patients' knowledge and experience of 'shielding', their experience of accessing both dialysis and general medical care during the pandemic, and their feelings about the pandemic and the future more generally (see supplementary material S1). Questionnaires were primarily sent out by post, but were also collected at renal clinic appointments and opportunistically during medical 
admissions. We received 31 responses during our data collection period of approximately 1 month, from a cohort of roughly 85 patients receiving PD. Although our questionnaire has not been validated, we are not aware of any validated PREMs relating to COVID-19, and these tools are commonly and successfully used to capture patient experience in a wide range of specialties. ${ }^{6}$

\section{Results}

While the majority of respondents to our questionnaire felt that their dialysis experience did not change during the COVID-19 pandemic, several other concerning repercussions were apparent from the data. Individuals with end-stage renal failure are classed as 'clinically extremely vulnerable' or 'high risk' by the NHS, and should have been advised by letter to shield to reduce their likelihood of contracting COVID-19. The term 'shielding' was understood by $90 \%$ of our respondents, and the majority recalled having received written advice about this from the NHS. Attending hospital was the most commonly cited reason for these patients being unable to shield effectively, with $55 \%$ needing to leave their house to attend a hospital appointment or to seek medical care. Some needed to leave the house for food shopping, and a minority travelled to work or went out for exercise. Although patients on PD do not attend a dialysis unit two or three times a week as with those undergoing $\mathrm{HD}$, this finding reflects the reality that they still make frequent trips to hospital for blood tests, clinic visits and PD tube changes. They are also unfortunately hospitalised not infrequently as a result of comorbidity, or occasionally related to issues with their dialysis. This comes with the risk of hospital-acquired coronavirus infection, which could have serious consequences in this vulnerable group.

Multiple patients on PD told us that they had become unwell during the pandemic (not necessarily with coronavirus), and four patients (13\%) reported experiencing difficulties accessing medical assistance at that time. Of note, many of those who became unwell contacted the renal unit directly for advice or for assessment. This reflects the significant role played by the renal unit in terms of the medical needs of a patient receiving PD, and the fact that the nephrologist of a patient undergoing dialysis may be their most frequent care provider. Nephrologists frequently look after multiple other aspects of their patients' health, for example blood pressure and cardiovascular risk, in addition to their kidneys. There is also a culture of placing patients at the centre of their own care, for example by empowering them to contact renal units directly when they are unwell, or by providing online access to their latest blood test results to enable them to monitor their disease. This positive relationship is unusual within the NHS and is perhaps even more important during the pandemic. It was also noted in the free text comments of the questionnaire by several respondents, who were grateful for the support of the renal unit during a difficult period (see supplementary material S2 for examples).

\section{Discussion}

The negative effects on the physical health of these vulnerable patients, particularly if they contract COVID-19, are not surprising given their unfortunate susceptibility to infections and other serious complications. However, perhaps the most concerning finding from our data was that $60 \%$ of respondents indicated that they feel negatively, or feel ambivalent, about the future. We know that the mental health of the general population has suffered significantly during the pandemic, with worrying increases in levels of mental distress in the UK. ${ }^{8}$ While the negative feelings reported in our survey are likely to be multifactorial for many patients, the isolation required during shielding and the health anxiety associated with being part of a high-risk group almost certainly play a significant role. It is also pertinent to remember that patients on the renal transplant list are currently facing even more uncertainty in the wake of a restricted transplant programme, which further adds to their mental stressors. We do not have any pre-pandemic data for direct comparison of the mental state of patients receiving PD. However, our centre participates in the Renal Association's yearly Kidney PREM, and it is notable that patients on home-based treatments including PD returned generally lower experience scores than those on in-centre HD in the 2020 report. ${ }^{9}$ This may suggest a wider pattern of patients on home-based renal therapies having struggled more than those who had frequent direct contact with renal centres during the pandemic.

Although this questionnaire-based study is limited in its breadth given the small number of respondents, it represents a local view from a centre which has a sizeable population on PD in an area badly affected by the COVID-19 pandemic. Despite this limitation, we feel that our findings highlight the key role that the renal multidisciplinary team can, and perhaps should, play for patients on PD during any subsequent waves of the pandemic. We feel that nephrology centres should take into account the significant impact that the pandemic continues to have on patients receiving either PD or HD, and aim to negate some of the avoidable potential harms to them. Ideally, contact with large healthcare centres, which may represent a vector for infection, should be replaced by phone or video consultations, and even home visits (for example for blood tests) wherever safe and practicable. National guidance is available to direct this. During clinical reviews, an inquiry into the patient's mood and general wellbeing could be invaluable, with signposting to online or telephone support services if a patient is found to be struggling. Although this may usually be undertaken by GPs, and in the knowledge that secondary care is currently under significant pressure, supporting our patients' mental health may become integral to our role as physicians given these extraordinary times. As members of the renal team have a unique and often close relationship with those in their care undergoing dialysis, this could be an opportunity.to provide vital holistic care at what is certainly a difficult time for our patients. More broadly, we hope that all physicians can apply these principles to the care of their patients with chronic diseases in subsequent pandemics, particularly those on home-based treatments who may otherwise be trying to cope alone.

\section{Supplementary material}

Additional supplementary material may be found in the online version of this article at www.rcpjournals.org/clinmedicine: S1 - PD patients' COVID-19 experience questionnaire. S2 - Sample of questionnaire responses.

\section{References}

1 Hsu C, Weiner D. COVID-19 in dialysis patients: outlasting and outsmarting a pandemic. Kidney Int 2020;98:1402-4.

2 UK Renal Registry. COVID-19 surveillance report for renal centres in the UK: A summary of the first wave of the pandemic March to August 2020. Bristol, UK: The Renal Association, 2020. 
https://renal.org/sites/renal.org/files/covid_report_first_wave_ FINAL_041220.pdf [Accessed August 2021].

3 GOV.UK. Coronavirus (COVID-19) in the UK. www.coronavirus.data. gov.uk [Accessed March 2021].

4 Kato S, Chmielewski M, Honda $\mathrm{H}$ et al. Aspects of immune dysfunction in end-stage renal disease. Clin J Am Soc Nephrol 2008;3:1526-33.

5 UK Renal Registry. UK Renal Registry 22nd Annual Report. Bristol, UK: The Renal Association, 2020. www.renal.org/audit-research/ annual-report [Accessed March 2021].

6 Kingsley C, Patel S. Patient-reported outcome measures and patientreported experience measures. BJA Education 2017;17:137-44.

7 NHS (2020). Who is at higher risk from coronavirus. www.nhs.uk/ conditions/coronavirus-covid-19/people-at-higher-risk/whos-athigher-risk-from-coronavirus [Accessed February 2021].
8 Pierce M, Hope H, Ford T et al. Mental health before and during the COVID-19 pandemic: a longitudinal probability sample survey of the UK population. Lancet Psychiatry 2020;7:883-92.

9 Renal Association. Patient reported experience of kidney care in the UK 2020. Bristol, UK: The Renal Association, 2020. https://renal. org/sites/renal.org/files/KQuIP/PREM \% 20report \% 202021.pdf [Accessed May 2021].

Address for correspondence: Dr Laura Balson, Heartlands Hospital, Bordesley Green East, Bordesley Green, Birmingham B9 5SS, UK.

Email: laura.balson@nhs.net 